\title{
Zentrale Faktoren bei der Umsetzung von Usability-Engineering bei einem mittelständischen Softwarehersteller in der Pflegebranche
}

Christoph Trappe ${ }^{1}$, Wilko Heuten ${ }^{1}$, Susanne Boll ${ }^{1}$, Simon Timmermanns ${ }^{2}$, Stefan Rahner ${ }^{3}$, Dietmar Wolff ${ }^{4}$, Britta Gräfe ${ }^{4}$

OFFIS - Institut für Informatik ${ }^{1}$

Johanniter-Unfall-Hilfe e.V. ${ }^{2}$

Softwareentwicklung, TARGIS GmbH ${ }^{3}$

FINSOZ e.V. ${ }^{4}$

\section{Zusammenfassung}

In diesem Beitrag geht es um die Identifikation von Faktoren, die die Integration von Methoden des Usability-Engineerings in den Softwareentwicklungsprozess beeinflussen. Diese werden am Beispiel eines mittelständischen Softwareherstellers in der Pflegebranche entlang des Prozesses zur Gestaltung gebrauchstauglicher interaktiver Systeme (ISO 9241-210:2010) dargestellt. Dabei gilt es, Hinweise für Kompetenzzentren zu sammeln und so Möglichkeiten eines dedizierten und für den Kontext adaptierten Angebotes für KMUs zu schaffen. Fazit des Beitrags ist, dass nicht nur Sinn und Zweck von Methoden des Usability-Engineerings, ihre phasenweise Zuordnung und Durchführung in statischen Methodenbeschreibungen deutlich gemacht werden müssen, sondern ein besonderes Augenmerk auf die nachhaltige Integration der Methoden in die Unternehmensstruktur gelegt werden muss. Dazu gehören Hinweise zur methodischen Auswertung und Gewinnung praxisrelevanter Erkenntnisse sowie Formate zur Kommunikation der Ergebnisse innerhalb des Unternehmens.

\section{$1 \quad$ Einleitung}

Im Jahr 2013 lebten in Deutschland 2,48 Millionen registrierte Pflegebedürftige. Bis zum Jahr 2030 werden es voraussichtlich 3,31 Millionen Menschen sein (Bundesministerium für Arbeit und Soziales 2015). Die Sozialwirtschaft steht angesichts der kontinuierlich steigenden Zahl von zu pflegenden Menschen und des knapper werdenden Pflegepersonals 
vor einer wachsenden Herausforderung, die Versorgung zu sichern. Der demografische Wandel hat demnach eklatante Auswirkungen auf die gesamte Sozialwirtschaft (Bundesministerium für Gesundheit 2015).

Für die Zukunft gilt es, die Effizienz der Arbeitsabläufe und die Attraktivität der Arbeitsbedingungen in dieser Branche zu verbessern. Insbesondere Zulieferer für die Sozialwirtschaft und Sozialverwaltung stehen in der Pflicht, angemessene Produkte für den Pflegesektor bereitzustellen, damit die Qualität der Pflege weiterhin sichergestellt oder sogar gesteigert werden kann. Hier spielt die intuitive Bedienbarkeit von Software eine wesentliche Rolle. Die Hersteller in der Sozialwirtschaft sind vorwiegend klein- und mittelständische Unternehmen mit guten Fachkenntnissen über die Domäne, die sich aber entweder den Vorteilen einer Steigerung der Gebrauchstauglichkeit nicht bewusst sind oder denen geprüfte Handlungsempfehlungen für konkrete Umsetzungsmaßnahmen fehlen (Woywode et al. 2011). Eine nachhaltige Breitenwirkung in der Schaffung eines Bewusstseins und konkreter Handlungsempfehlungen zum Thema Usability in der Pflegebranche und deren Verbreitung konnte bisher nicht erzielt werden. Dieser Umstand soll mit dem Projekt UCARE behoben werden.

\section{Methode}

In einem iterativen Vorgehen werden im Projekt UCARE Werkzeuge und Methoden für Softwareentwicklungs-KMUs in der Pflegebranche geschaffen und durch den Einsatz in der Praxis erprobt. In der ersten Iteration lieferte eine Analyse der beim KMU bestehenden Softwareentwicklungsprozesse, die als erste Grundlage für die Auswahl und Bewertung von Methoden diente, Einsichten zur gegenwärtigen Nutzereinbindung und zu Informationsflüssen im Unternehmen. Auf der Grundlage von kontextuellen Beobachtungen, Interviews und Befragungen beim KMU (Teammeetings, Treffen zur Produktkoordination und bilateralen Runden) sowie Informationen aus unternehmensbezogener Literatur konnten Erkenntnisse zur Unternehmensstruktur und den Abläufen bei der Softwareentwicklung generiert werden (Trappe \& Timmermanns 2014).

In Veranstaltungen im Workshop-Format und Präsentationen wurde eine Sensibilisierung für das Thema Usability im KMU vorangetrieben, ein gemeinsames Verständnis der Begrifflichkeiten „Gebrauchstauglichkeit“ und „Interaktive Systeme“ entwickelt und anhand der Frage „Wie werden interaktive Systeme bedienbar?" aus wissenschaftlicher und praktischer Perspektive diskutiert. Beispielsweise wurden Erhebungen mittels Interviews, Fragebögen, Fokusgruppen und ethnographischer Studien, wie Beobachtungen mit Video, Ton oder Sensorik thematisiert. Zudem wurden Tagebuchstudien, auf Grundlage derer sich z.B. Szenarien und Personas als Spezifikationen von Nutzungskontext und Anforderungen aus Nutzersicht erstellen lassen, als Grundlage der ersten Iteration der Entwicklung im Rahmen des Projektes vorgestellt. Anhand des Prozesses zur Gestaltung gebrauchstauglicher interaktiver Systeme konnte der mögliche Ablauf einer nutzerzentrierten Entwicklung dargestellt, ein ausgewähltes Methodeninventar für die unterschiedlichen Phasen der Entwicklung zusammengestellt und gemeinsam mit einem KMU erarbeitet werden. Die 
ausgewählten Methoden waren Shadowing, Interview, Persona, Szenario, Pro \& Contra Analyse, Fokusgruppen, Prototyping mit Softwaretools, Interaktionstoolkit und Lautes Denken. Alle Methoden wurden mittels Fragebogen, Beobachtungen oder qualitativen Aussagen hinsichtlich ihrer Praxistauglichkeit bewertet. So konnten wichtige Hinweise für die Angemessenheit der Methoden im Unternehmen gewonnen werden. Eine Vorstellung der Evaluation einiger der Methoden wurde an anderer Stelle festgehalten (Timmermanns et al. 2015). Die wichtigsten Erkenntnisse werden im Folgenden vorgestellt. Im Anschluss werden Hinweise für ein entsprechendes Beratungsangebot zusammengefasst.

\section{Ergebnisse: Faktoren der Integration entlang des HCD-Prozesses}

Die Bewertungen der einzelnen Methoden mittels Fragebogen, Beobachtungen oder qualitativen Aussagen sowie des Gesamtprozesses einer Iteration wurden gesammelt und hinsichtlich ihrer Praxistauglichkeit auf Schlüsselfaktoren bei der KMU-Integration begutachtet. Durch die Zusammenstellung in Clustern konnten eine Reihe von Themenfeldern identifiziert werden.

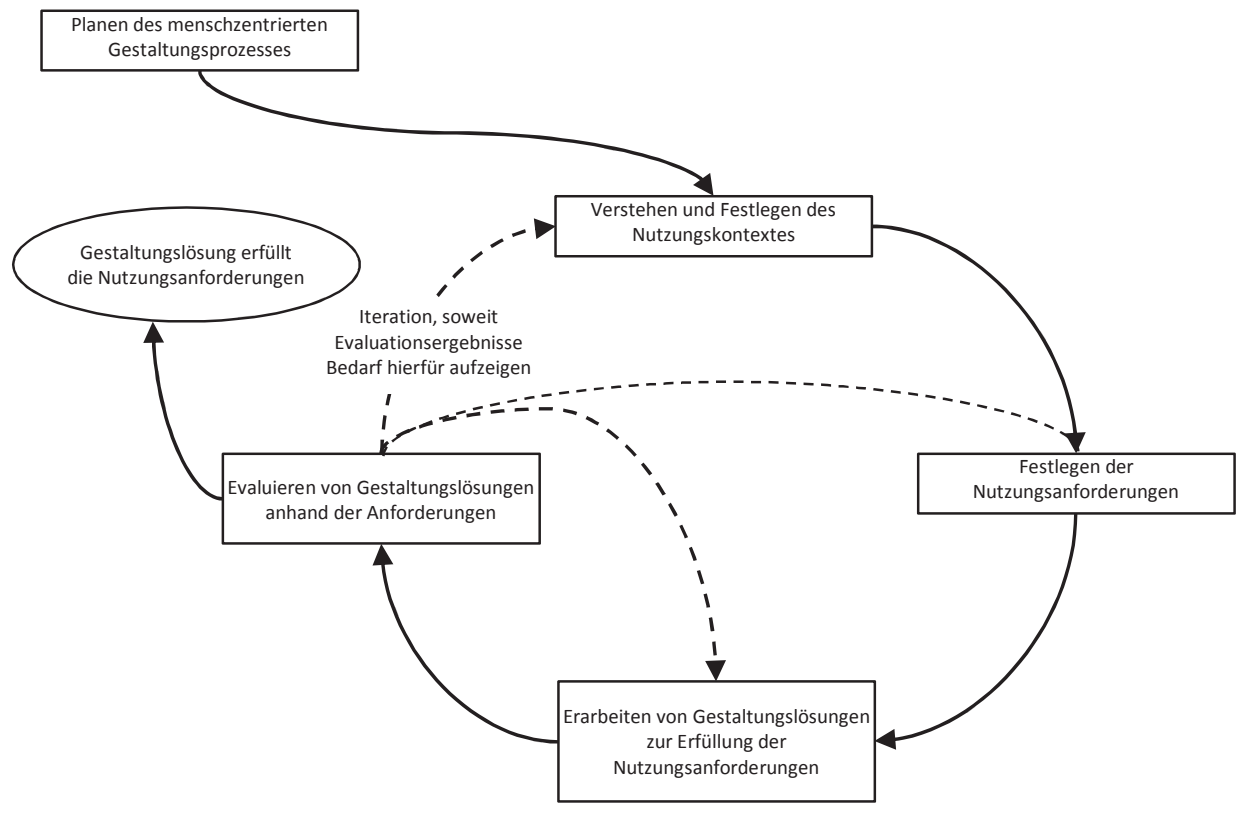

Abbildung 1: HCD-Prozess nach ISO 9241-210:2010

Im Folgenden werden Faktoren, die bei der Integration von Methoden des UsabilityEngineerings im KMU identifiziert wurden, entlang des Prozesses zur Gestaltung 
gebrauchstauglicher interaktiver Systeme (siehe Abbildung 1) bzw. des Vorgehens nach dem Human-Centred Design (HCD, siehe International Organization for Standardization 2010) vorgestellt.

Verantwortlichkeit klären: „Usability“ als abstraktes Oberthema wird als wichtiger Aspekt der Softwareentwicklung von weiten Teilen der Belegschaft, auch in unterschiedlichen Funktionen, erkannt. In der Planungsphase zeigt sich, dass die Vergabe von Verantwortlichkeit für das Thema an dedizierte Mitarbeiter explizit vorgenommen werden sollte.

Ressourcen bereitstellen: Eine Vergabe von Verantwortlichkeit für Usability im KMU sollte mit dem Zugeständnis von Ressourcen einhergehen. Zu beobachten ist, dass über die Freigabe von Ressourcen im KMU aus wirtschaftlicher Perspektive und nicht auf der Grundlage von Erkenntnisinteresse entschieden wird, was eine Barriere darstellt. Zwar gibt es Möglichkeiten, den Aufwand für Nutzerzentriertes Design effizient zu gestalten, doch gerade bei der Einführung entsteht Mehraufwand. Der langfristige Wettbewerbsvorteil scheint nicht unmittelbar ersichtlich. Hier lohnt es sich, Entscheidungsträger durch eine direkte Demonstration der Durchführung einer Methode von der Dringlichkeit der Maßnahmen zu überzeugen.

Gemeinsames Verständnis schaffen: Die Terminologie muss vorgestellt und erarbeitet werden, damit ein gemeinsames Verständnis von Usability im Unternehmen wachsen kann. Auffällig ist zum einen, dass die Thematik „Usability“ von den Mitarbeitern des KMU im Wesentlichen in der Evaluationsphase eines Produktes verortet wird und zum anderen sich auf vorwiegend funktionale Aspekte beschränkt und konzeptionelle Probleme hinsichtlich der Gebrauchstauglichkeit nicht betrachtet werden. Dies sollte bei zukünftigen Beratungsangeboten berücksichtigt werden.

Offiziellen Rahmen geben: Anfängliche Begeisterung für das Thema ohne offizielle Verankerung führen zu einer „Zwischen-Tür-und-Angel-Usability“, die nicht nachhaltig ist. Ist ein einzelner Verantwortlicher auch ein wichtiger Faktor für die Integration von Usability-Engineering in das KMU, so muss doch die gesamte Unternehmenskultur mit Usability-Kompetenzen wachsen und in interdisziplinären Runden spezifisch entwickelt werden, damit Ergebnisse beispielsweise von Nutzerstudien auch in der Entwicklung ankommen oder technische Vorgaben und Einschränkungen durch historisch gewachsene Software bei der Konzeption von Papierprototypen berücksichtigt werden.

Zeit effizient nutzen: Der Zeitfaktor bei der methodischen Erkenntnisgewinnung ist wesentlich mitentscheidend, ob eine Usability-Methode im KMU langfristig verwendet wird oder nicht. Dies sollte bei der Konzeption von Methoden, aber auch bei der Ausarbeitung von Usability-Leitfäden berücksichtigt werden.

Anforderungen individuell erfassen: Usability ist einfach, lässt sich in kleinem Umfang realisieren, muss jedoch auch in den Entwicklungsprozess des KMU fest verankert werden. Bei externen Beratungsangeboten müssen die Bedarfe des Unternehmens individuell abgefragt und Methoden ggf. kontextabhängig adaptiert werden. 
Ergebnisse verwerten: Vorgestellte Methoden des Usability-Engineerings werden zum Großteil als verständlich und nicht zu komplex bewertet. Gerade in der Beobachtung der Umsetzung zeigt sich, dass Methodenbeschreibungen auch Hinweise auf die Auswertung der Ergebnisse liefern sollten und wie diese innerhalb des Unternehmens kommuniziert werden können.

Entscheidungen validieren: Durch Nutzer verifizierte Aussagen können Produktmanager eine Argumentationsgrundlage für oder wider bestimmter Designentscheidungen liefern.

Kontakte aufbauen und vernetzen: Der Aufwand bei der Akquise von Endnutzern kann, abhängig von der Zielgruppe, die Bereitschaft des KMUs zur Investition von Ressourcen übersteigen. Gerade bei Pflegekräften, die in einem engen Zeitkorridor navigieren, kann die Bereitschaft zur Teilnahme an Nutzerstudien niedrig sein. Hier kann ein branchenspezifisches Kompetenzzentrum mit Kontakten $\mathrm{zu}$ Pflegediensten und Domänenexperten wertvolle Hinweise und Angebote liefern.

Praxisrelevanz schaffen: Es gibt eine Bereitschaft zur Investition von Ressourcen, wenn absehbar ist, dass der Einsatz einer Methode direkten Nutzen bringt und eine unmittelbare Praxisrelevanz hat. Somit müssen Beispiele und Leitfäden gegeben werden, die dicht am Tagesgeschäft der Mitarbeiter liegen. Es sollten an die Alltagsrealität angepasste Leitfäden geliefert werden, welche die unterschiedlichen Rollen in KMU mit zielgruppenspezifischer Ansprache adressieren. Dies ist eine der Herausforderungen an das Angebot eines Kompetenzzentrums.

Leitfäden liefern: Usability-Heuristiken sollten für den KMU-Einsatz aufbereitet und zur Verfügung gestellt werden. Auch wenn Usability-Verantwortliche im Unternehmen keine ausgebildeten Usability-Experten sind, kann man durch entsprechend vorbereitete Heuristiken eine Sensibilisierung für zentrale Aspekte von Gebrauchstauglichkeit bereitstellen. Auch die Planung und Ausarbeitung von Erhebungsinstrumenten kann durch wesentliche formalisierbare Usability-Eigenschaften zielgerichtet angepasst werden.

Die Identifikation von Faktoren, welche die Integration von Methoden des UsabilityEngineerings in den Softwareentwicklungsprozess erschweren, legen einige Hinweise für das Kompetenzzentrum nahe, die im folgenden Fazit zusammengefasst werden.

\section{$4 \quad$ Fazit}

Ein Kompetenzzentrum sollte ein breit gefächertes Beratungsangebot liefern - von der Hilfestellung bei der Planung, über Probandenakquise und Auswertung der Methoden bis hin zur nachhaltigen Integration der Ergebnisse in den Arbeitsablauf des KMU. Zu einem Teil ist die Integration von Usabiliy-Engineering in den Entwicklungsprozess stets abhängig von den Eigenheiten eines Unternehmens. Doch gibt es eine große Chance, branchenspezifisch das Bewusstsein für Usability $\mathrm{zu}$ schärfen und mit konkreten, fachspezifischen Handlungsempfehlungen die Unternehmen ansprechen zu können. Die Landschaft der Hersteller von Software für Pflegedienstleistungen in Deutschland ist durch viele klein- und 
mittelständige Unternehmen gekennzeichnet, die einen Bedarf an einheitlichen, standardisierten Usability-Konzepten haben. Viele dieser Unternehmen sind zwar in spezifischen Verbänden organisiert, das Schaffen von Bewusstsein und konkreten Handlungsempfehlungen zum Thema Usability in der Pflegebranche und deren Verbreitung spielt bisher jedoch eine untergeordnete Rolle. Eine Umfrage, die gegenwärtig in Planung ist, soll dazu beitragen, dass Verständnis der Branche $\mathrm{zu}$ vertiefen und ein klareres Bild der Branche im Hinblick auf die Skalierbarkeit von praxistauglichen UUX-Methoden und Erkenntnissen zeichnen zu können.

\section{Referenzen}

\section{Über das Projekt}

Das Projekt UCARE ist Teil der Förderinitiative „Einfach intuitiv - Usability für den Mittelstand“, die im Rahmen des Förderschwerpunkts „Mittelstand-Digital - IKTAnwendungen in der Wirtschaft" vom Bundesministerium für Wirtschaft und Energie (BMWi) gefördert wird. Der Förderschwerpunkt unterstützt gezielt kleine und mittlere Unternehmen (KMU) sowie das Handwerk bei der Entwicklung und Nutzung moderner Informations- und Kommunikationstechnologien (IKT). „Mittelstand-Digital“ setzt sich zusammen aus den Förderinitiativen ,eKompetenz-Netzwerk für Unternehmen“ mit 38 eBusiness-Lotsen, „eStandards: Geschäftsprozesse standardisieren, Erfolg sichern“ mit derzeit 16 Förderprojekten und „Einfach intuitiv - Usability für den Mittelstand“ mit zurzeit 13 Förderprojekten. Weitere Informationen sind unter www.mittelstand-digital.de abrufbar.

\section{Literaturverzeichnis}

Bundesministerium für Arbeit und Soziales (2015). Fortschrittsbericht 2014 zum Fachkräftekonzept Der Bundesregierung. http://www.bmas.de/SharedDocs/Downloads/DE/PDF-Publikationen/ fortschrittsbericht-fachkraefte-fuer-2014.pdf?_blob=publicationFile.

Bundesministerium für Gesundheit (2015). Zahlen und Fakten zur Pflegeversicherung. http://www.bmg.bund.de/fileadmin/dateien/Downloads/Statistiken/Pflegeversicherung/Zahlen_und _Fakten/150601_Zahlen_und_Fakten_Pflegeversicherung_03-2015.pdf.

International Organization for Standardization (2010). ISO 9241-210:2010, Ergonomics of HumanSystem Interaction - Part 210: Human-Centred Design for Interactive Systems. Distributed through American National Standards Institute.

Timmermanns, S., C. Trappe, W. Heuten, et al. (2015) UCARE: Entwicklung eines Usability Kompetenzzentrums für fie Pflegebranche. In Proceedings 8. AAL-Kongress. VDE e. V., Hrsg.

Trappe, Christoph, and Simon Timmermanns (2014). Towards Best Practice Solutions: Usability Engineering Für Die Pflegebranche. In Workshop-Proceedings of the 2014 Conference on Humans and Computers. München, Deutschland.

Woywode, Michael, Alexander Mädche, Dieter Wallach, and Marcus Plach (2011) Gebrauchstauglichkeit von Anwendungssoftware Als Wettbewerbsfaktor Für Kleine Und Mittlere Unternehmen (KMU). http://www.usability-in-germany.de/studie. 